\title{
Portal flow steal after liver transplantation
}

\author{
Bohyun Kim1, Kyoung Won Kim², Gi-Won Song ${ }^{3}$, and Sung-Gyu Lee ${ }^{3}$ \\ 'Department of Radiology, Ajou University Medical Center, Suwon; '²epartment of Radiology, Asan Medical Center, University of Ulsan \\ College of Medicine, Seoul; ${ }^{3}$ Division of Liver Transplantation and Hepatobiliary Surgery, Department of Surgery, Asan Medical Center, \\ University of Ulsan College of Medicine, Seoul, Korea
}

Portal flow steal occasionally persists even after the liver transplantation, which may reduce the portal flow and thus threaten the patients' outcome. Therefore, pre- and peri-operative detection of portal steal phenomenon requiring radiological or surgical interruption is essential for the liver transplantation candidates as well as for the recipients. (Clin Mol Hepatol 2015;21:314-317)

Keywords: Liver transplantation; Portosystemic shunt; Portal flow steal; Doppler

\section{INTRODUCTION}

After liver transplantation, maintaining adequate portal flow secures nutrients and stimuli essential for the graft. Especially during the early postoperative period after partial graft liver transplantation, sufficient portal inflow is vital for boosting rapid liver regeneration. ${ }^{1}$ Portosystemic shunt is not uncommon in patients with portal hypertensive liver cirrhosis. While this kind of shunt may be shut down with normalization of portal pressure after the transplantation, occasionally, it lingers after the transplantation and continues to divert hepatopetal flow away from the graft. ${ }^{2}$ Since this shunt can potentially "steal" significant amount of portal inflow and even jeopardize the graft, timely detection of portal flow steal is crucial to patient outcome. In this article, we present imaging findings and percutaneous intervention management of portal flow steal in a recipient of partial graft liver transplantation.

\section{CASE}

A 38-year-old male patient, who had undergone living donor liver transplantation using a right lobe graft due to chronic hepatitis B associated liver cirrhosis, visited emergency department at our institution complaining of fatigue and pruritis. The operation had been uneventful, and the patient had been well after the liver transplantation. On physical examination, the patient had icteric sclera and yellow coloration of the skin. Blood chemistry revealed the following findings; hemoglobin, $13.7 \mathrm{~g} / \mathrm{dL}$; leukocyte count, $4,900 / \mathrm{mm}^{3}$; platelet count, $131,000 / \mathrm{mm}^{3}$; total protein $7.0 \mathrm{~g} / \mathrm{dL}$; albumin, $2.8 \mathrm{~g} / \mathrm{dL}$; total bilirubin, $19.7 \mathrm{mg} / \mathrm{dL}$; aspartate aminotransferase (AST), $553 \mathrm{IU} / \mathrm{L}$; alanine aminotransferase (ALT), 378 IU/L; gamma-glutamyltranspeptidase (GGT), 432 IU/L.

On Doppler ultrasound study, portal flow velocity was weakened to $18.8 \mathrm{~cm} / \mathrm{sec}$, with preserved hepatopetal directional flow (Fig. 1A). The hepatic arterial and hepatic venous Doppler parameters and waveforms were normal. Under the suspicion of portal flow steal, the tributaries of the portal vein were evaluated there-

\section{Abbreviations:}

AST, aspartate aminotransferase; ALT, alanine aminotransferase; SMV, superior mesenteric vein; IMV, inferior mesenteric vein

\section{Corresponding author: Kyoung Won Kim}

Department of Radiology, Asan Medical Center, University of Ulsan College of Medicine, 88 Olympic-ro 43-gil, Songpa-gu, Seoul 05505, Korea Tel: +82-2-3010-4385, Fax: +82-2-476-4719

E-mail: kimkw@amc.seoul.kr 

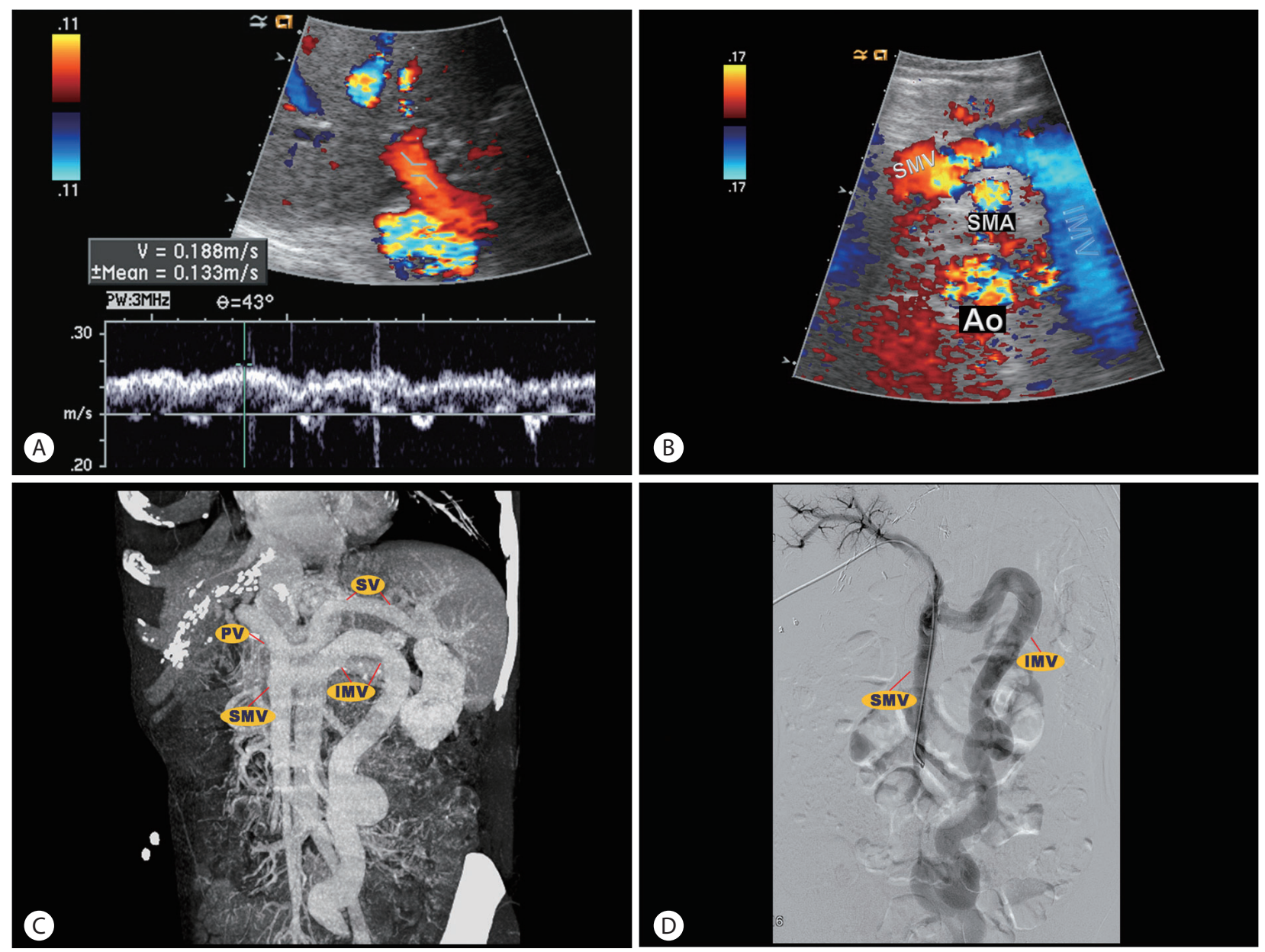

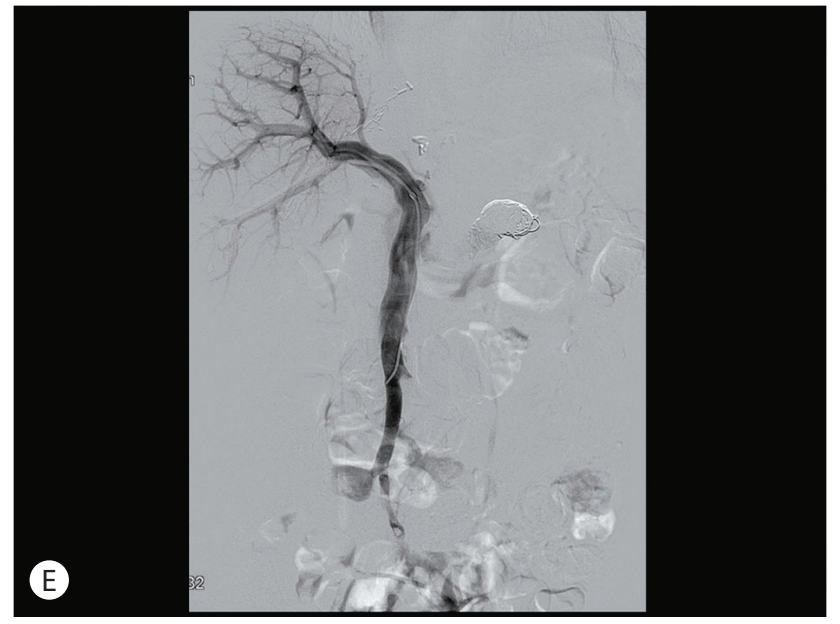

after. Upon inspecting the superior mesenteric vein (SMV) at the level just below the portomesenteric junction, abnormally dilated inferior mesenteric vein (IMV) inserting to the SMV was noticed. On color Doppler study, hepatofugal directional flow was evident, stealing the portal flow away from the graft (Fig. 1B). Subsequent $\mathrm{CT}$ revealed marked engorgement of the IMV, in line with the
Figure 1. Recurrent portal steal via the inferior mesenteric vein-rectal varix in 38-year-old male patient who underwent living donor liver transplantation for portal hypertensive liver cirrhosis. (A) Doppler spectrogram obtained at graft portal vein shows somewhat diminished portal flow, measuring $18.8 \mathrm{~cm} / \mathrm{s}$. (B) Color Doppler ultrasound at level just below portomesenteric junction demonstrates reversed flow via distended inferior mesenteric vein (IMV). (C) On maximum intensity projection image of portal venous phase, markedly distended IMV is intuitively visualized, which continues to rectal varix (not covered). (D) On direct portogram obtained by injection of contrast agent at distal part of superior mesenteric vein (SMV), contrast flow from the SMV is mostly shunting to the IMV, indicating the significant portal flow steal. (E) After the successful embolization of the IMV, portal flow is directed to the liver. SMV, superior mesenteric vein; SMA, superior mesenteric artery; $\mathrm{Ao}_{0}$ abdominal aorta; PV, portal vein; SV, splenic vein.

\section{Doppler ultrasound findings (Fig. 1C).}

In order to block the steal, percutaneous embolization was attempted. After percutaneous, transhepatic puncture of the right portal vein, guide wire and catheter was advanced to the SMV under the guidance of fluoroscopy. On portogram, portal flow from the SMV was mostly shunted to the IMV (Fig. 1D). After suc- 
cessfully embolizing the IMV using multiple coils and lipiodol, final portogram demonstrated no residual portal steal and all the flow from the SMV was directed to the graft (Fig. 1E). After the procedure, AST and ALT were decreased to $112 \mathrm{IU} / \mathrm{L}$ and $132 \mathrm{IU} / \mathrm{L}$, respectively.

\section{DISCUSSION}

In portal hypertensive liver cirrhosis, gradual rise of hepatic sinusoidal resistance leads to shunting of portal flow into systemic circulation via less resistive collateral vessels. Depending on the amount of shunt flow, portal inflow is diminished to various degrees. ${ }^{3}$ When there is a significant reduction of portal inflow resulting from portosystemic shunt, it is referred to as portal flow steal. ${ }^{4}$

Usually after liver transplantation using adequate-size graft, normalization of portal pressure shuts down the shunt and collapses the collateral vessels. However, major pathways of portosystemic shunts larger than $10 \mathrm{~mm}$ in diameter may not shrink. ${ }^{5}$ Also, consistently high intrahepatic vascular resistance resulting from acute rejection, volume overload, congestion, or small-forsize graft can hinder spontaneous regression of the shunt. ${ }^{6-9}$ Persistent portosystemic shunt may cause graft dysfunction by stealing portal inflow essential for the graft.' Continuous portal flow steal afflicts partial graft recipients more than whole-liver recipients, as partial graft is more vulnerable to portal venous hemodynamic changes while is in greater need for robust portal flow prompting quicker regeneration in order to meet the metabolic demand of the recipient. ${ }^{4}$ Therefore, prudent monitoring on portal inflow is necessary in recipient of partial graft liver transplantation.

Radiologically, patients with portal flow steal shows decreased portal flow velocity, and may even show bidirectional or reversed flow direction on Doppler ultrasound. ${ }^{2}$ While Doppler ultrasound is supreme in demonstrating flow direction and velocity, CT can visualize various collateral vessels at different location. In that regard, combination of Doppler ultrasound and CT helps detecting potential route of the portal flow steal. Percutaneous or direct portogram is used both for the diagnosis and treatment of the portal flow steal. After localizing the steal by studying the flow direction on portogram, the route of the steal can be obliterated either by embolization only or in conjunction with surgical ligation. Then, additional portogram can be performed to assess the completeness of the manipulation. ${ }^{10}$
In the current case, portal flow steal was first detected by Doppler ultrasound, and then the culprit collateral vessel was further exposed by CT. Finally, the steal was percutaneously embolized, using portogram as guidance. Our case exemplifies an ideal combination of radiologic studies in detecting and managing the steal.

In conclusion, radiologists should pay attention to the potential portal flow steal in the liver transplantation candidates and recipients. Meticulous inspection of serial changes in the Doppler parameters and timely utilization of $\mathrm{CT}$ and portogram help detect and treat devastating steal phenomenon.

\section{Conflicts of Interest}

The authors have no conflicts to disclose.

\section{REFERENCES}

1. Lee SG, Moon DB, Ahn CS, Kim KH, Hwang S, Park KM, et al. Ligation of left renal vein for large spontaneous splenorenal shunt to prevent portal flow steal in adult living donor liver transplantation. Transpl Int 2007;20:45-50.

2. Horrow MM, Phares MA, Viswanadhan N, Zaki R, Araya V, Ortiz J. Vascular steal of the portal vein after orthotopic liver transplant: Intraoperative sonographic diagnosis. J Ultrasound Med 2010;29:125128.

3. Wachsberg RH, Bahramipour P, Sofocleous CT, Barone A. Hepatofugal flow in the portal venous system: pathophysiology, imaging findings, and diagnostic pitfalls. RadioGraphics 2002;22:123-140.

4. Ikegami T, Shirabe K, Nakagawara H, Yoshizumi T, Toshima T, Soejima $Y$, et al. Obstructing spontaneous major shunt vessels is mandatory to keep adequate portal inflow in living-donor liver transplantation. Transplantation 2013;95:1270-1277.

5. Sanada Y, Mizuta K, Urahashi T, Wakiya T, Ihara Y, Okada N, et al. Impact of posttransplant portosystemic shunts on liver transplantation. World J Surg 2012;36:2449-2454.

6. Braun MM, Bar-Nathan N, Shaharabani E, Aizner S, Tur-Kaspa R, Belenky $A$, et al. Postshunt hepatic encephalopathy in liver transplant recipients. Transplantation 2009;87:734-739.

7. De Carlis L, Del Favero E, Rondinara G, Belli LS, Sansalone CV, Zani B, et al. The role of spontaneous portosystemic shunts in the course of orthotopic liver transplantation. Transpl Int 1992;5:9-14.

8. Duffy JP, Hong JC, Farmer DG, Ghobrial RM, Yersiz H, Hiatt JR, et al. Vascular complications of orthotopic liver transplantation: experience in more than 4,200 patients. J Am Coll Surg 2009;208:896903; discussion 903-895.

9. Sadamori H, Yagi T, Matsukawa H, Matsuda H, Shinoura S, Umeda 
Bohyun Kim, et al. Portal flow steal after liver transplantation

Y, et al. The outcome of living donor liver transplantation with prior spontaneous large portasystemic shunts. Transpl Int 2008;21:156162.

10. Kim JH, Ko GY, Sung KB, Yoon HK, Kim KR, Moon DB, et al. Trans- venous variceal embolization during or after living-donor liver transplantation to improve portal venous flow. J Vasc Interv Radiol 2009;20:1454-1459. 